INPLASY

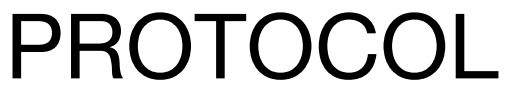

To cite: Li et al. Moxibustion therapy for treating patients with postpartum urinary retention A systematic review and meta-analysis protocol. Inplasy protocol 202140037. doi:

10.37766/inplasy2021.4.0037

Received: 07 April 2021

Published: 07 April 2021

Corresponding author:

Tianjiao Li

deresia@126.com

Author Affiliation:

Beijing University of Chinese

Medicine

Support: NNSFC No.81874503.

Review Stage at time of this submission: Preliminary

searches.

Conflicts of interest:

None declared.

\section{Moxibustion therapy for treating patients with postpartum urinary retention A systematic review and meta-analysis protocol}

\author{
Li, T1; Zhao, B²; Hui, X3; Wang, H4; Lin, Y5.
}

Review question / Objective: The aim of this review is to access the effectiveness and safety of moxibustion therapy for postpartum urinary retention.

Condition being studied: Postpartum urinary retention (PUR) is one of the most common complications after parturition. It refers to women cannot urinate spontaneously within 6-8 hours or incompletely urinate after giving birth. PUR may leads to abdominal pain, increasing vaginal haemorrhage, urinary tract infection, which prolong the length of hospitalization and increase the costs. Moxibustion is a viable supplemental replacement therapy in China, US, Japan and many other countries and regions. Years of application experience and clinical trials have shown that moxibustion can promote the recovery of postpartum urinary retention. Moxibustion is a non-invasive therapy, it has the advantages of lower costs, painless and less adverse reactions compared to medications and catheterization. This systematic review is aimed to evaluate whether moxibustion is a good choice for PUR patients, and whether it is as effective to be a supplement therapy to other therapies.

INPLASY registration number: This protocol was registered with the International Platform of Registered Systematic Review and Meta-Analysis Protocols (INPLASY) on 07 April 2021 and was last updated on 07 April 2021 (registration number INPLASY202140037).

\section{INTRODUCTION}

Review question / Objective: The aim of this review is to access the effectiveness and safety of moxibustion therapy for postpartum urinary retention.

Condition being studied: Postpartum urinary retention (PUR) is one of the most common complications after parturition. It refers to women cannot urinate spontaneously within 6-8 hours or incompletely urinate after giving birth. PUR may leads to abdominal pain, increasing vaginal haemorrhage, urinary tract infection, which prolong the length of hospitalization and increase the costs. Moxibustion is a viable supplemental 
replacement therapy in China, US, Japan and many other countries and regions. Years of application experience and clinical trials have shown that moxibustion can promote the recovery of postpartum urinary retention. Moxibustion is a noninvasive therapy, it has the advantages of lower costs, painless and less adverse reactions compared to medications and catheterization. This systematic review is aimed to evaluate whether moxibustion is a good choice for PUR patients, and whether it is as effective to be a supplement therapy to other therapies.

\section{METHODS}

Participant or population: Patients diagnosed with PUR after parturition will be included. There will be no restrictions on age, race, education, and economic status.

Intervention: Moxibustion therapy and related therapies will be included as interventions in this review, including moxa stick moxibustion, moxibustion with moxa cone, and moxibustion with moxibustioner (a device for moxibustion). Moxibustion combined with other therapies like acupuncture, massage, cupping, and drugs will be excluded.

Comparator: The following treatment comparisons will be investigated: 1 . Moxibustion compared with no treatment; 2. Moxibustion compared with placebo or sham moxibustion; 3. Moxibustion compared with other therapies.

Study designs to be included: We will include randomized controlled trials (RCTs) and randomized cross-over trials related with moxibustion in the treatment for PUR without restrictions on publication status. Quasi-randomized trials will be excluded.

Eligibility criteria: Two review authors (TL and $\mathrm{XH}$ ) will independently screen the titles, abstracts and keywords of all retrieved studies and decide which trials meet the inclusion criteria. We will obtain the full text of all studies of possible relevance for further assessment. Excluded studies will be recorded with reasons. We will independently extract data from the selected reports or studies and fill in the designed data extraction form. We will obtain data for general information, participants, methods, interventions, outcomes, results, adverse events, conflict of interest, ethical approval and other information. We will contact the authors for further information when the reported data is not sufficient. Any disagreement will be resolved by discussion between the two authors ( $T L$ and $X H$ ) and further disagreements will be arbitrated by the third author (BXZ).

Information sources: The following electronic databases will be searched, regardless of publication status and languages, from their respective inception dates to February 2021: the Cochrane Central Register of Controlled Trails (CENTRAL), PubMed, EMBASE, the Web of Science, China National Knowledge Infrastructure(CNKI), Chinese Biomedical Literature Database(CBM), Chinese Scientific Journal Database (VIP database), and Wan-Fang Database. Furthermore, we will search the conference abstracts and trial registered platforms, including ClinicalTrials.gov and the Chinese Clinical Trial Registry (ChiCTR) to obtain ongoing or unpublished trials. The reference lists and the citation lists of studies meeting the inclusion criteria and relevant systematic reviews will also be searched to identify further studies for inclusion. The original authors will be contacted for clarification when necessary.

Main outcome(s): The primary outcomes will be cure rates and post-void residual bladder volume. PVRV under ultrasonic testing is a widely accepted measure to evaluate the bladder function.

Additional outcome(s): The secondary outcomes will be total effective rates and first urination time. In addition, adverse events in the treatment will also be accessed in the review to evaluate the safety of moxibustion therapy.

Quality assessment / Risk of bias analysis: Two authors (HW and YL) will assess the 
risk of bias with the Cochrane Collaboration's tool for risk of bias assessment for all included studies. We will evaluate the following domains for risk of bias: sequence generation, allocation sequence concealment, blinding of participants and personnel and outcome assessors, incomplete outcome data, selective outcome reporting and other sources of bias. The assessments will be classified into three levels: low risk, high risk and unclear risk.

Strategy of data synthesis: Data synthesis will be conducted with the RevMan V.5.3. The results will be expressed as a risk ratio for dichotomous data and standardized mean difference for continuous data. If the $I^{2}$ test is less than $\mathbf{5 0 \%}$, the fixed effect model will be used for data synthesis. If the $I^{2}$ test is between $50 \%$ to $75 \%$, the randomeffects model will be conducted for data synthesis. And if the $I^{2}$ test is higher than $75 \%$, we will find the possible reasons from both clinical and methodological perspectives and provide an explanation or conduct subgroup analysis.

Subgroup analysis: None planned at present, but if the data are available, subgroup analyses will be conducted for different operative types and moxibustion methods. In addition, if significant heterogeneity is found to exist, we will also conduct a subgroup analysis, if necessary.

Sensitivity analysis: Sensitivity analysis will be conducted to evaluate the robustness of the primary results. The principal decision nodes will include methodological quality, sample size, and the effect of missing data. The meta-analysis will be repeated and studies of lower quality will be excluded.

Country(ies) involved: China.

Keywords: complementary medicine, moxibustion therapy, postpartum urinary retention, protocol, systematic review.

Contributions of each author:

Author 1 - Tianjiao Li.

Email: deresia@126.com

Author 2 - Baixiao Zhao.
Email: baixiao100@vip.sina.com

Author 3 - Xin Hui.

Author 4 - Hao Wang.

Author 5 - Yao Lin. 